\title{
TIME-FREQUENCY SIGNATURE RECONSTRUCTION FROM RANDOM OBSERVATIONS USING MULTIPLE MEASUREMENT VECTORS
}

\author{
Moeness G. Amin, Yimin D. Zhang, and Branka Jokanović \\ Center for Advanced Communications \\ Villanova University, Villanova, PA 19085, USA
}

\begin{abstract}
A new approach for sparse nonstationary signal reconstruction based on multiple windows is introduced. Signals which are localizable in the time-frequency (TF) domain give rise to sparsity in the same domain. When combined, sparse reconstructions, applied to randomly sampled data and corresponding to different selected windows, provide enhanced TF signature estimation. Among possible orthogonal windows, we consider those which characterize the eigen-decomposition of reducedinterference quadratic time-frequency distribution kernels. The highly overlapping TF support of the windows' full-data spectrograms inspires the use of the multiple measurement vectors, in lieu of individual windowed signal recovery. It is shown that the proposed approach outperforms other reconstruction methods when only a single window is applied and is superior to reduced interference time-frequency distributions of random observations.
\end{abstract}

Index Terms - Time-frequency distribution, multiple measurement vector, compressive sensing, random sampling

\section{INTRODUCTION}

Nonstationary signals arise in a broad class of active sensing modalities, including sonar, radar, and ultrasound. They also characterize many passive sensing problems such as speech and electromyographic recordings. Time-frequency signal representations (TFSRs) enable separations of nonstationary signals that are mixed in both time and frequency domains [1-4]. Windowing and filtering based approaches do not allow the capture and separation of individual nonstationary signal components. TFSRs are mainly obtained using quadratic timefrequency distributions (QTFDs) which have their roots in the nonparametric Wigner-Ville distribution (WVD).

Owing to their power concentrations over the joint timefrequency (TF) variables, the signatures of a large class of nonstationary signals occupy small regions in the TF domain. This property casts the signals as sparse in the joint-variable representations. As such, it invites sparse signal reconstruction and compressive sensing techniques $[5,6]$ to play an important role in revealing the time-frequency signal analysis and processing of single- and multi-component signals [7-12].

In this paper, we consider QTFDs applied to randomly sampled signals where the averaging sampling frequency is below Nyquist. We focus on reduced-interference distribution (RID) kernels which act on eliminating, or at least considerably attenuating, the cross-terms. These interference terms are highly pronounced in WVD and tend to clutter and obscure the true signal power concentrations signified by the auto-terms $[13,14]$.

QTFDs typically face challenges in implementation stemming from the need to perform convolution operations, at each sample, followed by the Fourier transform (FT). This motivated the representations of QTFD as a combination of spectrograms, which are both positive and amenable to fast FT computations. The spectrogram, or squared-magnitude of the sliding-window short-time Fourier transform (STFT), is the principal tool used to estimate the time-dependent spectral energy density in many applications. While there are many ways and criteria guiding the selection of the spectrogram windows [15-21], we deal, without loss of generality, with those which are obtained as eigenvectors of desirable timefrequency distribution (TFD) kernels, an approach known as spectrogram decompositions of TFDs [22, 23].

In this paper, we perform sparse reconstruction of TFSRs from random time-domain samples using multiple windows, reminiscent of the multiple window spectrograms and spectrogram decompositions of TFDs. In so doing, we avoid any smoothing or convolution operations and operate directly on the data. Further, the reconstructions corresponding to the different windows can be averaged to provide an improved TFSR over a single window-based reconstruction. The overlapping nature of the different window reconstructions amounts to a common sparse support property and inspires the use of multiple measurement vectors (MMV) techniques within the compressive sensing paradigm. This enables achieving enhanced signal localization in the TF domain over single measurement vectors (SMVs) corresponding to individually reconstructions. The MMV problem is solved using the complex multitask Bayesian compressive sensing method.

In Section 2 of this paper, we motivate the problem and show examples of possible orthogonal windows for use in SMV and MMV TF reconstruction. Section 3 discusses the MMV reconstruction using Bayesian compressive sensing techniques, and Section 4 includes computer simulations, covering different frequency modulated (FM) signals and showing results corresponding to observations with full and missing data samples.

\section{MOTIVATION AND SIGNAL MODEL}

In classical nonparametric spectral analysis and estimation, multi-window FTs and their respective square magnitudes, 
known as periodograms, bear the advantage, when averaged, over a single-window based FT in providing reduced variance and stable estimates. These windows were chosen to possess particular characteristics such as orthogonality and optimality within certain signal bandwidth. The same hold true in nonstationary signal analysis and processing. It was shown that multiple windows outperform single-window spectrograms in terms of localization and power concentration properties in the TF domain. One approach was to approximate QTFDs by a weighted sum of spectrograms. The result enjoys the fast computations of the fast FT (FFT) and the high cross-term attenuation of the RIDs. This was achieved by eigendecomposition of a rotated RID kernel and considering only the eigenvalues and eigenvectors of the dominant components [22, 23].

The Cohen's class of discrete-time TFD of a signal $x(t)$, when characterized by an ambiguity-domain kernel $\phi(\theta, \tau)$, is expressed as

$$
C_{x}(t, \omega)=\sum_{\theta} \sum_{\tau} \sum_{u} \phi(\theta, \tau) x(u+\tau) x^{*}(u-\tau) e^{j(\theta u-\omega \tau-\theta t)},
$$

where $\theta$ is the Doppler shift and $\tau$ is the delay. Define the autocorrelation-domain kernel as the FT of the ambiguity-domain kernel with respect to $\theta$, expressed as

$$
\psi(t, \tau)=\sum_{\theta} \phi(\theta, \tau) e^{-j \theta t},
$$

which, for a real-valued TFD with a real-valued ambiguity domain kernel, is conjugate-symmetric in both $t$ and $\tau$ [23]. Using the unit-Jacobian linear transformation of variables

$$
\left[\begin{array}{l}
t_{1} \\
t_{2}
\end{array}\right]=\left[\begin{array}{cc}
1 & \frac{1}{2} \\
1 & -\frac{1}{2}
\end{array}\right]\left[\begin{array}{l}
t \\
\tau
\end{array}\right],
$$

we can denote the rotated autocorrelation-domain kernel as

$$
\tilde{\psi}\left(t_{1}, t_{2}\right)=\psi\left(-\frac{t_{1}+t_{2}}{2}, t_{1}-t_{2}\right) \text {. }
$$

Define $\boldsymbol{\Psi}$ as a matrix that contains $\tilde{\psi}\left(t_{1}, \mathrm{t}_{2}\right)$ for all values of $t_{1}$ and $t_{2}$. Then, its eigen-decomposition leads to

$$
\boldsymbol{\Psi}=\sum_{k=1}^{L} \lambda_{k} \mathbf{e}_{k} \mathbf{e}_{k}^{H},
$$

where $L$ is the rank of $\boldsymbol{\Psi}$, whereas $\lambda_{k}$ and $\mathbf{e}_{k}$ are the nonzero eigenvalues and the respective eigenvectors. In this case, the TFD $C_{x}(t, \omega)$ can be expressed in the following form [23]:

$$
\begin{aligned}
C_{x}(t, \omega) & =\sum_{k=1}^{L} \lambda_{k}\left|\sum_{n=0}^{N-1} x(t+n) e_{k}^{*}(n) e^{-j \omega(t+n)}\right|^{2} \\
& =\sum_{k=1}^{L} \lambda_{k}\left|\sum_{n=0}^{N-1} x(t+n) e_{k}^{*}(n) e^{-j \omega n}\right|^{2},
\end{aligned}
$$

where $e_{k}(n)$ is the $n$th element of $\mathbf{e}_{k}$. As such, the TFD is obtained as the weighted sum of the spectrograms, each is generated by the magnitude square of the FT of the data weighted by one of the eigenvectors of the rotated kernel. In practice, the eigen-decomposition of $\boldsymbol{\Psi}$ can be well approximated by a small number $\left(L_{0} \leq L\right)$ of dominant terms, yielding fast RID reconstruction.

As an example, Figs. 1 and 2 show the eigenvalues and the six dominant eigenvectors of Choi-Williams kernel, which is given in the ambiguity domain as [13]

$$
\phi(\theta, \tau)=e^{-\theta^{2} \tau^{2} / \sigma},
$$

where $\sigma$ is a parameter determining the kernel support in the ambiguity domain. $\sigma=100$ is used to generate the figures. In addition, time and frequency smoothing Hanning windows, respectively of lengths 15 and 123, are applied. It is clear that the eigenvalues decay very fast, and those after the 6th term are negligible. As such, six terms are considered in the sequel for a high-fidelity TFD reconstruction. Note that the eigendecompositions are data-independent and thus can be computed only once prior to TFD reconstructions.

As an example, consider a noise-free signal which consists of two components, one sinusoid and one chirp. The signal waveform, the spectrogram and the CWD are shown in Fig. 3, where a Hanning window of 63 is used in computing the spectrogram. The CWD is computed using the same parameters mentioned above. The sinusoidal frequency is 0.1 , whereas the initial and end frequencies of the chirp are 0.2 and 0.45 , respectively. The spectrograms corresponding to the six eigenvectors, which respectively multiply the observed data, are shown in Fig. 4. The eigenvectors act as modulators to generate a single or multiple components around the true timefrequency signatures. Their sum, weighted by the respective eigenvalues, is shown in Fig. 5(a). Clearly the combined spectrogram is superior to each of the six spectrograms acting alone. The positive and negative eigenvalues allow the multiple spectrograms to interplay such that the weighted sum has better localization signal properties.

It is important to note that only the dominant eigenvectors can closely approximate the RID TFD with a small number of terms. As a counterexample, we show in Fig. 5(b) the sum of the six terms that correspond to the least significant eigenvalues. The results are highly cluttered and do not represent the proper TF power distribution of the data. This underscores the importance of proper selections of the multiple windows for enhanced TFD.

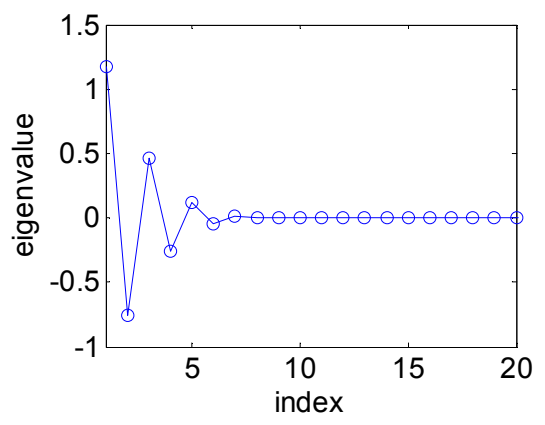

Fig. 1. The first 20 dominant terms of the eigenvalue of the rotated autocorrelation-domain kernel, sorted by the magnitude. 
eig vec 1
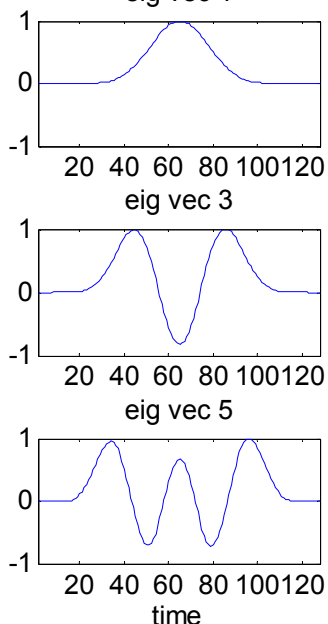

Fig. 2. Eigenvectors of the first six dominant terms.
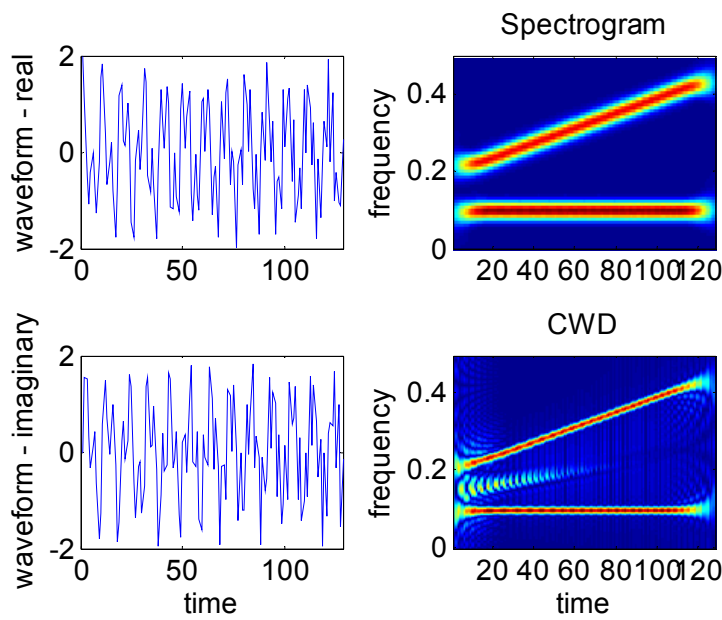

Fig. 3. Signal waveform with its Spectrogram and CWD.

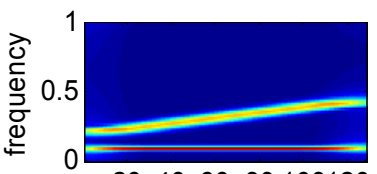

20406080100120

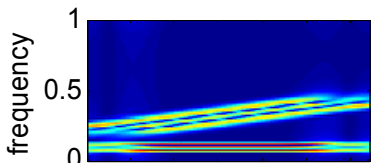

20406080100120

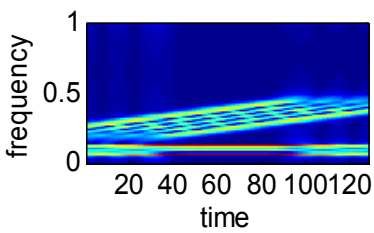

eig vec 2

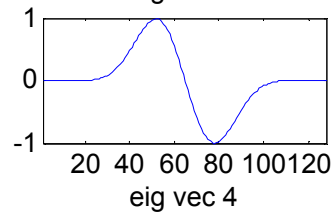

eig vec 6

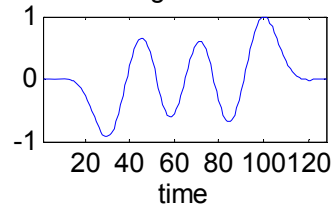

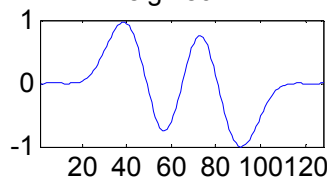

\section{TFD RECONSTRUCTION EXPLOITING MULTIPLE-MEASUREMENT VECTOR}

Inspired by the multiple window spectrograms and motivated by the fact that the local signal behavior of multicomponent FM signals has a sparse TF representation, as evident from Fig. 4, we apply multiple windows for FM signal construction from few random observations. The windows are selected to be the same as those in Fig. 2 resulting from eigen-decomposition of TF RID kernels. Other window choices and kernels are possible and can be motivated by different arguments [24].

For the convenience of notation, we express the STFT inside the magnitude-square in (6), for each $t$, in a vector format as

$$
\mathbf{s}_{k}(t)=\tilde{\mathbf{F}} \mathbf{y}_{k}(t), k=1, \ldots, L,
$$

where the $i$ th element of $\mathbf{s}_{k}(t)$ is $\left[\mathbf{s}_{k}(t)\right]_{i}=x(t+i) e_{k}^{*}(i), \tilde{\mathbf{F}}$ is the inverse FT matrix, and $\mathbf{y}_{k}(t)$ is the $k$ th column of the TFD matrix to be reconstructed. Note that the rows corresponding to the missing data are removed from $\mathbf{s}_{k}(t)$ and $\tilde{\mathbf{F}}$ for each $t$. Expression (8) represents a SMV when solved for each $k$ separately. It can also define a MMV model, exploiting the approximately common support of $\mathbf{y}_{k}(t)$ in the TF domain for different $k$. This characteristic is also referred to as the block sparsity or group sparsity, which can be effectively solved using techniques that account for such property. Group sparsity solvers include block-sparsity based compressed sensing [25], multi-task compressed sensing [26, 27], and distributed compressed sensing [28].

The Bayesian based compressive sensing algorithms, which are based on the relevance vector machine (RVM), generally achieves better reconstruction performance than others. In this approach, $\mathbf{y}_{k}(t)$ is treated as weight vector whose $m$ th element is drawn from the following zero-mean Gaussian distribution:

$$
y_{k, m}(t) \sim N\left(x_{k, m}(t) \mid 0, \alpha_{m}(t)\right),
$$

where $\alpha_{m}(t)$ is the variance of the Gaussian probability density function. The group sparsity of $\mathbf{y}_{k}(t)$ over different values of $k$ is considered by forcing $\alpha_{m}(t)$ to be shared by the $L$ measurements (windows). Multi-task Bayesian compressing sensing techniques effectively solve the distribution of the sparse entries as well as the noise characteristics through iterations [26, 27].

The Bayesian algorithms have originally been designed to recover real-valued sparse solutions [26]. An effective extension to complex problems is proposed in [27] to recover complex signals in the MMV model. Therefore, in this paper, the complex multitask Bayesian compressive sensing method [27] is used.

\section{SIMULATION RESULTS}

Simulations results are provided to demonstrate the effectiveness of the proposed multiple window sparse reconstruction of

Fig. 4. The spectrogram of the six components. 
TF signatures of nonstationary signals. Both SVMs and MMVbased sparse reconstruction techniques are applied and compared. In the former, the TFD is separately reconstructed using the SMV for each window $k$ in (8), and the results are weighted with the respective eigenvalues and then averaged.

The TFSR based on the MMV is shown in Fig. 6(a), whereas that corresponding to the weighted sum of the SMV results is shown in Fig. 6(b). The data considered is all observations of the two-component signal described previously. Both results provide accurate reconstructions with insignificant cross-terms, with the MMV results depicting slightly better localization at the two ends.

Now we consider a case where only $25 \%$ random timedomain samples (i.e., 32 out of the 128 samples) of the data are available. All other parameters remain unchanged. Fig. 7 shows the observed signal waveform as well its spectrogram and CWD. In this case, neither the spectrogram nor the CWD shows clear TF signatures. The TFSR obtained from the MMV model through the multi-task Bayesian algorithm yields much cleaner results, as evident in Fig. 8(a), whereas the weighted sum of the individually reconstructed TFD results, as depicted in Fig. 8(b), shows inferior TF signatures and a high level of undesired artifacts. For comparison, the sparse reconstruction result when using a single 64-point Hanning window is depicted in Fig. 8(c), which does not show a clear signature of the chirp component.

\section{CONCLUSION}

Wideband frequency modulated (FM) signals are sparse in frequency when considered locally and, as such, can be reconstructed from random observations. The paper showed that sparse reconstruction can benefit from applying multiple windows to the data prior to solving the underdetermined linear equations with the dictionary made of Fourier atoms. We applied a set of windows which are the eigenvectors of reduced-interference distribution kernels. We demonstrated the advantages of both combining the reconstruction results corresponding to the different multiple-measurement vectors (MMVs) and the simultaneous reconstruction using MMVs. The proposed approach was shown to outperform timefrequency distributions using spectrograms and reducedinterference distributions when applied to random observations of multi-component FM signals. It was also shown that the proposed approach is superior to reconstruction based on only a single window.

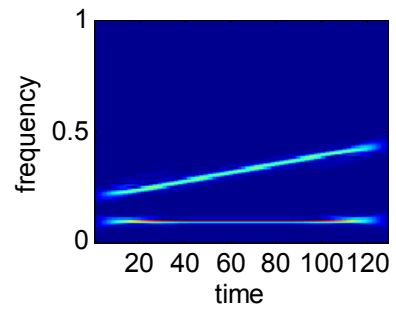

(a)

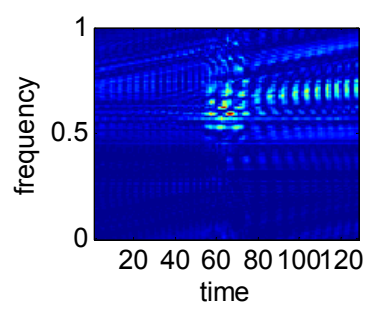

(b)
Fig. 5. (a) Sum of the six dominant component spectrograms. (b) Sum of the six least significant component spectrograms.

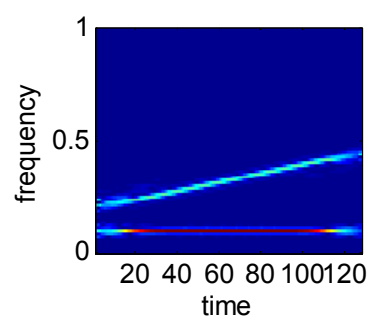

(a)

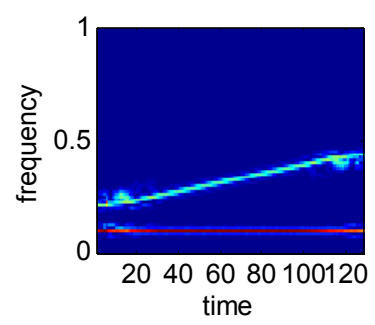

(b)
Fig. 6. (a) TFD reconstructed from MMV; (b) Sum of the TFDs individually reconstructed from the respective SMV.
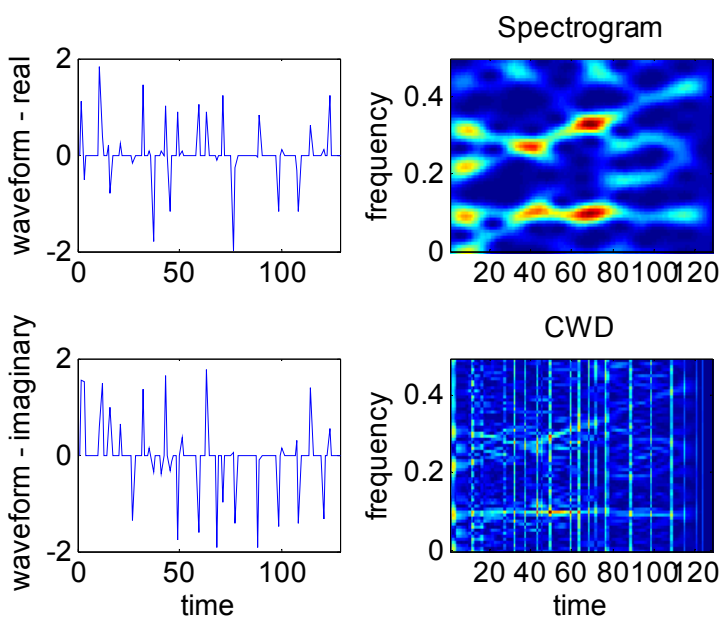

Fig. 7. Signal waveform of $25 \%$ observation data with its Spectrogram and CWD.

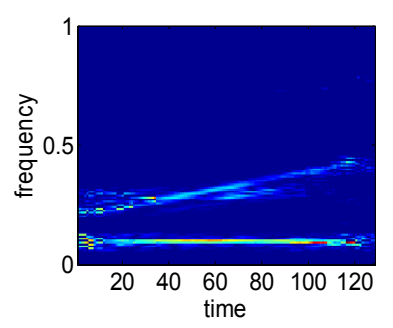

(a)

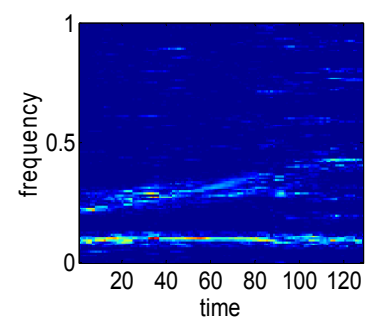

(b)

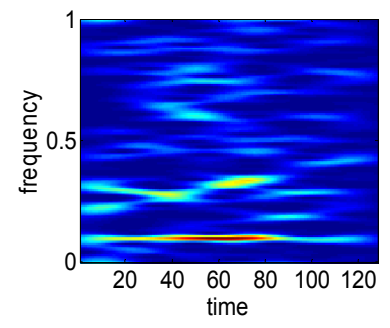

(c)

Fig. 8. TFD reconstructed using $25 \%$ of observed data. (a) sparse reconstruction from MMV approach; (b) sum of the six individually reconstructed component TFD; and (c) sparse reconstruction using a single rectangular window. 


\section{REFERENCES}

[1] P. Flandrin, Time-Frequency/Time-Scale Analysis. New York, NY: Academic, 1999.

[2] B. Boashash, Time Frequency Signal Analysis and Processing. New York, NY: Elsevier, 2003.

[3] L. Cohen, "Time-frequency distributions- A review," Proc. IEEE, vol.77, no.7, pp. 941-981, July 1989.

[4] P. Flandrin, M. Amin, S. McLaughlin, and B. Torresani, (Eds.), special issue on Time-Frequency Analysis and Applications, IEEE Signal Proc. Mag., Nov. 2013.

[5] J. Candes and M. Wakin, "An introduction to compressive sampling," IEEE Signal Proc. Mag., vol. 25, no. 2, pp. 2130, March 2008.

[6] D. L. Donoho, "Compressed sensing," IEEE Trans. Inform. Theory, vol.52, no.4, pp.1289-1306, April 2006

[7] P. Flandrin and P. Borgnat, "Time-frequency energy distributions meet compressed sensing," IEEE Trans. Signal Proc., vol. 58, no. 6, pp. 2974-2982, June 2010.

[8] Y. D. Zhang and M. G. Amin, "Compressive sensing in nonstationary array processing using bilinear transforms," in Proc. IEEE Sensor Array and Multichannel Signal Proc. Workshop, Hoboken, NJ, June 2012.

[9] LJ. Stankovic, S. Stankovic, I. Orovic, and M. G. Amin, "Robust time-frequency analysis based on the L-estimation and compressive sensing," IEEE Signal Proc. Lett., vol. 20, no. 5, pp. 499-502, May 2013.

[10] LJ. Stankovic, I. Orovic, S. Stankovic, and M. G. Amin, "Compressive sensing based separation of nonstationary and stationary signals overlapping in time-frequency," IEEE Trans. Signal Proc., vol. 61, no. 18, pp. 4562-4572, Sept. 2013.

[11] Y. D. Zhang, M. G. Amin, and B. Himed, "Reduced interference time-frequency representations and sparse reconstruction of undersampled data," in Proc. European Signal Proc. Conf., Marrakech, Morocco, Sept. 2013.

[12] B. Jokanović, M. Amin, and S. Stanković, "Instantaneous frequency and time-frequency signature estimation using compressive sensing," in Proc. SPIE Defense, Security and Sensing, May 2013.

[13] H. Choi and W. J. Williams, "Improved time-frequency representation of multicomponent signals using exponential kernels," IEEE Trans. Acoust., Speech, Signal Proc, vol. 37, no. 6, pp. 862-871, June 1989.

[14] W. Williams and J. Jeong, "Reduced interference timefrequency distributions," in B. Boashash (Ed.), Methods and Applications of Time-Frequency Signal Analysis, Melbourne, Australia: Longman Cheshire, 1992.
[15] G. Frazer and B. Boashash, "Multiple window spectrogram and time-frequency distributions," in Proc. IEEE ICASSP, pp. 293-296, April 1994.

[16] M. Bayram and R. G. Baraniuk, "Multiple window timefrequency analysis," in Proc. IEEE-SP Int. Sym. TimeFrequency and Tame-Scale Analysis, pp.173-176, June 1996.

[17] J. W. Pitton, "Time-frequency spectrum estimation: An adaptive multitaper method," in Proc. IEEE-SP Int. Sym. Time-Frequency and Time-Scale Analysis, pp. 665-668, Oct. 1998.

[18] F. Cakrak and P. Loughlin, "Multiple window nonlinear time-varying spectral analysis," in Proc. IEEE ICASSP, pp. 2409-2412, May 1998.

[19] W. J. Williams and S. Aviyente, "Optimal window timefrequency distribution decompositions," in Proc. Asilomar Conf. Signals, Systems and Computers, pp.817-821, Nov. 1998.

[20] S. Aviyente and W. Williams, "Multitaper reduced interference distribution," in Proc. IEEE Workshop on Statistical Signal and Array Proc., Pocono Manor, PA, Aug. 2000.

[21] M. Hansson, "Multiple window decomposition of timefrequency kernels using a penalty function for suppressed sidelobes," in Proc. IEEE ICASSP, Toulouse, France, May 2006.

[22] M. G. Amin, "Spectral decomposition of time-frequency distribution kernels," IEEE Trans. Signal Proc., vol. 42, no. 5, pp. 1156-1165, May 1994

[23] G. S. Cunningham and W. J. Williams, "Fast implementations of generalized discrete time-frequency distributions," IEEE Trans. Signal Proc., vol. 42, no. 6, pp. 1496-1508, June 1994.

[24] S. B. Hearon, M. G. Amin, "Minimum-variance timefrequency distribution kernels," IEEE Trans. Signal Proc, vol. 43, no. 5, pp. 1258-1262, May 1995

[25] Y. C. Eldar, P. Kuppinger, and H. Bolcskei, "Compressed sensing of block-sparse signals: uncertainty relations and efficient recovery," IEEE Trans. Signal Proc., vol. 58, no. 6, pp. 3042-3054, 2010.

[26] S. Ji, D. Dunson, and L. Carin, "Multitask compressive sensing," IEEE Trans. Signal Proc., vol.57, no.1, pp.92-106, 2009.

[27] Q. Wu, Y. D. Zhang, M. G. Amin, and B. Himed, "Complex multitask Bayesian compressive sensing," in Proc. IEEE ICASSP, Florence, Italy, May 2014.

[28] B. Dror, B. W. Michael, F. D. Marco, S. Shriram, and G. B. Richard, "Distributed compressed sensing of jointly sparse signals," in Proc. Asilomar Conf. Signals, Systems and Computers, Pacific Grove, CA, pp.1537-1541, Nov. 2005. 